\title{
Automated Fiber Type Identification in SDN-Enabled Optical Networks
}

\author{
Emmanuel Seve ${ }^{\circledR}$, Jelena Pesic (), Member, IEEE, Camille Delezoide ${ }^{(1)}$, Alessio Giorgetti ${ }^{(1)}$, \\ Andrea Sgambelluri ${ }^{\circledR}$, Nicola Sambo ${ }^{\circledR}$, Sébastien Bigo ${ }^{\left({ }^{\circ}\right.}$, Fellow, IEEE, \\ and Yvan Pointurier (1), Senior Member, IEEE
}

(Highly-Scored Paper)

\begin{abstract}
Network design margins are introduced by quality of transmission estimator inaccuracies. Some of those inaccuracies are due to uncertainty on the fiber type deployed in optical networks, and on the value of the chromatic dispersion of deployed fibers. We propose, in this paper, a method to identify all unknown fiber types (and estimate chromatic dispersion) in an optical network to reduce said uncertainties. We monitor, collect, centralize, and correlate chromatic dispersion values accumulated over each established light path, already measured by coherent receivers to autonomously identify fiber types without traffic interruption. Thanks to this method, we can identify the fiber type of all the 41-unknown links for a 28-node network built with four different fiber types and only 100 light paths. Our method is also capable of distinguishing two fiber types that only differ by their dispersion slope. We have also deployed and demonstrated this fiber type identification method at the ECOC 2018 demo session with real-time communication with our Lab from the demo floor. The SDN-enabled optical network is based on ONOS controller that periodically provides real-time chromatic dispersion values of active light paths needed for the autonomous fiber type identification.
\end{abstract}

Index Terms-Chromatic dispersion, fiber type, margin, monitoring, network, SDN.

\section{INTRODUCTION}

$\mathbf{T}$ O REACH the ultimate grail where the optical network operates close to its maximum capabilities, operators need to decrease their "design margins" [2], [3]. Various machine learning-based techniques [4]-[14] were proposed to decrease design margins. In [4]-[9], different machine learning methods have been developed to numerically show how the estimation of the Quality of Transmission (QoT) can be improved

Manuscript received October 15, 2018; revised December 26, 2018; accepted January 22, 2019. Date of publication January 29, 2019; date of current version April 2, 2019. This work was supported by the H2020 EU project ORCHESTRA under Grant 645360. This paper was presented in part at the 44th European Conference on Optical Communication, Roma, Italy, September 23-27, 2018. (Corresponding author: Emmanuel Seve.)

E. Seve, J. Pesic, C. Delezoide, S. Bigo, and Y. Pointurier are with Nokia Bell Labs, Nozay 91620, France (e-mail: emmanuel.seve@ nokia-bell-labs.com; jelena.pesic@nokia-bell-labs.com; camille.delezoide@nokia-bell-labs.com; sebastien.bigo@nokia-bell-labs.com; yvan.pointurier@nokia-bell-labs.com).

A. Giorgetti, A. Sgambelluri, and N. Sambo are with Scuola Superiore Sant'Anna, Pisa 56127, Italy, and also with CNIT, Pisa 56127, Italy (e-mail: alessio.giorgetti@santannapisa.it; andrea.sgambelluri@sssup.it; n.sambo@sssup.it).

Color versions of one or more of the figures in this paper are available online at http://ieeexplore.ieee.org.

Digital Object Identifier 10.1109/JLT.2019.2896041 for new traffic demands by monitoring network parameters. We can either improve the existing QoT model by decreasing the uncertainty on the network parameters via a hybrid machinelearning approach [4], or build a new model using classical machine-learning approaches [5]-[9]. For example, the approach used in [7], [8] is based on a Random Forest classifier, method fed by a set of network features (i.e., number of links, total length, longest link length, traffic volume and modulation format) and the output is a binary variable indicating whether the bit-error-rate is lower than the system threshold. In [9] a method based on a Gaussian Process regression was considered to predict the bit-error-rate using the signal power, the number of spans, the baud rate and the channel spacing as features. Experiments have been also conducted in [10]-[14] with real network testbeds. The first experimental testbed is based on a 6-node network and open ROADMs that constantly monitor performance of established demands [10]-[12]. The accuracy of the signal-to-noise ratio (SNR) estimation for new light paths was improved by learning the SNR of each link composing the light path. In [13], [14], the field performance is learned using artificial neural networks.

In this paper, among all sources of uncertainties, we focus on inaccurate fiber type deployed in a network and on chromatic dispersion. The fiber type is an essential input parameter provided by operators to system vendors at the earliest stage of system design. Owing to outdated records or erroneous fiber connections, it might happen that an operator uses inaccurate fiber types, particularly when buying or renting a part of the network infrastructure from another operator. This situation occurs increasingly often, as non-telecom companies (e.g., stadium operators) build their own infrastructures.

\section{A. Current Solutions}

Usually a full Fiber Optic Characterization (FOC) service is done several weeks before network commissioning. The following individual tests are done on a span-by-span basis: bidirectional optical time domain reflectometer shots, end-to-end loss with a power meter insertion test and chromatic dispersion test meter which provides accumulated chromatic dispersion and slope over the span. This process is still performed manually and consists of sending two technicians at neighboring sites to perform the tests. Due to complexity, a good FOC team can 


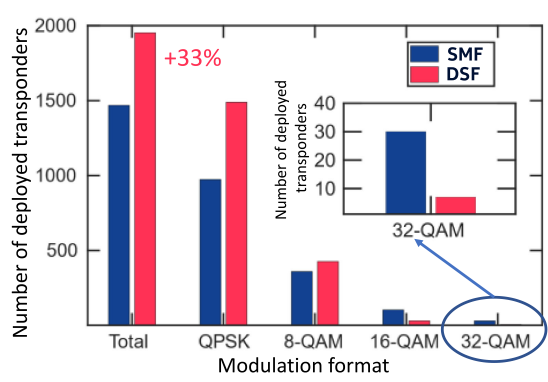

(a)

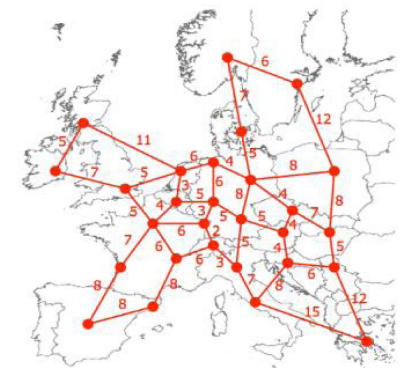

(b)

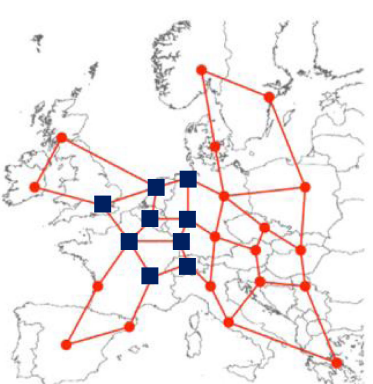

(c)

Fig. 1. (a) Number of deployed transponders in case of SMF-based and LEAF-based European topology. (b) The European backbone network topology consisting of $N=28$ nodes, 41 chromatic dispersion uncompensated bidirectional links. (c) Part of the European backbone network topology consisting of $N=9$ nodes, 12 chromatic dispersion uncompensated bidirectional links.

measure on average 2 to 3 spans a day. This process is extremely time-consuming.

To avoid this operational expenditure (OPEX), one solution for operators is to allocate a design margin, leading to additional capital expenditure (CAPEX) through over-dimensioning. Indeed, when fiber characteristics are missing or coming from an unreliable source, the network is designed by considering that all unknown fibers belong to the worst fiber type in terms of propagation impairments (selected among a diversity of fiber types known to be deployed in the same network [15]). This is leading to increased design margins and network design over-dimensioning by under-estimation of signal reaches. An evaluation in terms of number of transponders to be deployed in a network for two different types of fibers will be presented in Section II.

\section{B. New Solution: Paper Contribution}

In Section III, we propose and describe a technique based on Mixed Integer Linear Programming (MILP). This technique autonomously and simultaneously determines the fiber type and chromatic dispersion by correlating the chromatic dispersion over all established network light paths measured by deployed coherent receivers. We show in Section IV that, for a 41-links/28-node network, $98 \%$ of the network links are correctly identified even with a $400 \mathrm{ps} / \mathrm{nm}$ chromatic dispersion measurement uncertainty. Increasing the knowledge of the network in terms of fiber types allows to better estimate the QoT for the new traffic demands and thereby reduce design margins. A worst-case network dimensioning with SMF/LEAF fibers can lead to up to $2 \mathrm{~dB}$ design margin due to $6 \mathrm{~dB}$ difference for nonlinear noise variance between SMF and LEAF fibers [16]. In addition, exact knowledge of the chromatic dispersion parameters (absolute value and slope) is particularly critical for Dispersion Shifted Fiber (DSF) fibers where the nonlinear impairments can strongly vary according to the exact zero-dispersion wavelength and an even larger design margin should be considered with such fibers. In Section V, we demonstrate this identification method in an SDN-enabled optical network, where an ONOS controller has been properly extended to periodically provide the real time chromatic dispersion value of active light paths to the MILP-based method.

\section{NETWORK DIMENSIONNING: IMPACT OF THE FIBER TYPE}

To assess the benefits of having the knowledge of the exact type of the fiber deployed in the field, we perform network
TABLE I

FibER DisPERSION CHARACTERISTICS FROM DATA SHEETS (@ $\lambda_{0}=1550 \mathrm{~nm}$ )

\begin{tabular}{ccccc}
\hline \hline & DSF & LEAF & TL & SMF \\
\hline$D(\mathrm{ps} / \mathrm{nm} / \mathrm{km})$ & 0 & 4.2 & 8 & 16.7 \\
$D^{\prime}\left(\mathrm{ps} / \mathrm{nm}^{2} / \mathrm{km}\right)$ & 0.07 & 0.084 & 0.04 & 0.057 \\
$\Delta D\left(\mathrm{ps} / \mathrm{nm}^{2} / \mathrm{km}\right)$ & 0.3 & 0.3 & 0.3 & 0.5 \\
$\Delta D^{\prime}\left(\mathrm{ps} / \mathrm{nm}^{2} / \mathrm{km}\right)$ & $1 \mathrm{e}-3$ & $1 \mathrm{e}-3$ & $1 \mathrm{e}-3$ & $1 \mathrm{e}-3$ \\
\hline \hline
\end{tabular}

TABLE II

REACH

\begin{tabular}{cccc}
\hline \hline $\begin{array}{c}\text { Channel } \\
\text { Modulation }\end{array}$ & Data Rate & $\begin{array}{c}\text { SMF } \\
(\mathrm{km})\end{array}$ & $\begin{array}{c}\text { LEAF } \\
(\mathrm{km})\end{array}$ \\
\hline PDM-QPSK & $100 \mathrm{~Gb} / \mathrm{s}$ & 3120 & 2080 \\
PDM-8QAM & $150 \mathrm{~Gb} / \mathrm{s}$ & 1520 & 960 \\
PDM-16QAM & $200 \mathrm{~Gb} / \mathrm{s}$ & 640 & 400 \\
PDM-32QAM & $250 \mathrm{~Gb} / \mathrm{s}$ & 400 & 240 \\
\hline \hline
\end{tabular}

studies on the European topology (topology description is given in Fig. 1(b): 41 links and 28 nodes. We compare the number of transponders needed to fulfill traffic demands in the case of a European topology consisting of either the SMF-28 or the LEAF fiber from Corning (replaced and simplified respectively by SMF and LEAF in the following). The fiber characteristics are listed in Table I.

We consider uniform distribution of traffic demands between all nodes with 20 random draws and capacity ranging from $100 \mathrm{~Gb} / \mathrm{s}$ to $1 \mathrm{~Tb} / \mathrm{s}$ with steps of $100 \mathrm{~Gb} / \mathrm{s}$. We stop each simulation when reaching $1 \%$ blocking of the total demanded capacity. We assume elastic optical transponders are used to establish the light paths, working at 32 GBaud, supporting the following 4 modulation formats: polarization division multiplexed (PDM)QPSK, 8-QAM, 16-QAM and 32-QAM. Each modulation has its own reach as listed in Table II [17]. To allow the network to always work at optimal power, the launch power at span input is set to $0 \mathrm{dBm}$ for SMF fiber links (between two nodes) and $-2 \mathrm{dBm}$ for LEAF fiber links made of $80 \mathrm{~km}$-long spans (a link consists of several spans) and scaled accordingly for shorter spans [18]. Results presented in Fig. 1(a) show a 33\% increase of the number of deployed elastic transponders in case of LEAF-based topology compared to SMF-based topology. This increase in the number of transponders directly translates into higher network cost (CAPEX) and higher investments for an operator. 


\section{MILP-BASEd METHOd DESCRIPTION}

From the fiber vendors catalogs, it can be concluded that the fiber type can be fully assessed from chromatic dispersion parameters (dispersion and dispersion slope), which are rarely measured per link - this would incur traffic interruption, as mentioned above - but at best per light path (between a transmitter and a receiver). By correlating accumulated chromatic dispersion measurements for several (established) light paths, we simultaneously infer the fiber type and the chromatic dispersion parameters for each link. As accumulated chromatic dispersion is measured at different wavelengths, we also estimate the dispersion slope; the zero-dispersion wavelength could be determined from the latter estimation.

While the network is operational, for each new traffic demand we allocate a route and a wavelength, i.e., a light path. However, the technique proposed in this paper is independent of the specific routing and wavelength/spectrum allocation algorithm.

The parameters of optical networks are never perfectly known. In this work, we consider uncertainties on link lengths, on all fiber chromatic dispersion possible values (including dispersion slope), and on the measured accumulated chromatic dispersion for each established light path.

For all parameters and equations, we use the following acronyms:

\section{A. Acronyms}

D: chromatic dispersion, CD: accumulated chromatic dispersion, LP: light path, L: link

All variables and parameters of our system can be divided in two categories: input and output parameters, as listed below:

\section{B. Input Parameters}

- $N^{L P}$ : number of light paths;

- $N$ : number of network links;

- $\lambda_{0}$ : central wavelength $(1550 \mathrm{~nm})$;

- $\lambda_{j}$ : wavelength of light path $j$;

- $\triangle C D^{L P}: \mathrm{CD}$ measurement uncertainty;

- $\Theta_{i, j}:$ (binary) $=1$ if link $i$ is on light path $j$;

- $C D_{j, \text { meas }}^{L P}(\lambda)$ : measured CD for light path $j$;

- $C D_{i}^{L}\left(\lambda_{0}\right)_{k, \text { min }}$ : minimum CD for link $i$ assuming fiber type $k$, see (5);

- $C D_{i}^{L}\left(\lambda_{0}\right)_{k, \text { max }}$ : maximum CD for link $i$ assuming fiber type $k$, see (6);

- $D\left(\lambda_{0}\right)_{k}$ : chromatic dispersion at $\lambda_{0}$ for fiber type $k$;

- $D^{\prime}\left(\lambda_{0}\right)_{k}$ : chromatic dispersion slope at $\lambda_{0}$ for fiber type $k$;

- $L_{i}$ : length of the link $i$ according to data sheet;

- $\Delta D_{k}$ : chromatic dispersion range for fiber type $k$;

- $\Delta D_{k}^{\prime}$ : chromatic dispersion slope range for fiber type $k$;

- $\Delta L$ : link length uncertainty;

- $N_{f}$ : number of possible fiber types.

\section{Output Parameters}

- $C D_{i}^{L}\left(\lambda_{0}\right)$ : CD of the link $i$ for $\lambda_{0}$;

- $C D_{i}^{\prime L}\left(\lambda_{0}\right)$ : CD slope of the link $i$ for $\lambda_{0}$;

- $F T_{i, k}$ : fiber type (=1 if link $i$ is type $k$ );
The possible range for the accumulated chromatic dispersion of the light path $j$ is then expressed by Eq. (1)-(2):

$C D_{j, \text { meas }}^{L P}(\lambda)-\Delta C D^{L P} \leq \sum_{i=1}^{N}\left[\begin{array}{c}C D_{i}^{L}\left(\lambda_{0}\right) \\ +\left(\lambda-\lambda_{0}\right) C D_{i}^{\prime L}\left(\lambda_{0}\right)\end{array}\right] \Theta_{i, j}$

$\sum_{i=1}^{N}\left[\begin{array}{c}C D_{i}^{L}\left(\lambda_{0}\right) \\ +\left(\lambda-\lambda_{0}\right) C D_{i}^{\prime L}\left(\lambda_{0}\right)\end{array}\right] \Theta_{i, j} \leq C D_{j, \text { meas }}^{L P}(\lambda)+\Delta C D^{L P}$

Where $C D_{j, \text { meas }}^{L P}(\lambda)-\Delta C D^{L P}, C D_{j, \text { meas }}^{L P}(\lambda)+\Delta C D^{L P}$ are respectively the minimal and maximal possible values for the link-accumulated chromatic dispersion due to the measurement uncertainty $\triangle C D^{L P}$

The possible range for the accumulated chromatic dispersion of the link $i, C D_{i}^{L}\left(\lambda_{0}\right)$ is expressed by Eq. (3)-(4):

$$
\begin{aligned}
& \sum_{k=1}^{N_{f}} C D_{i}^{L}\left(\lambda_{0}\right)_{k, \min } F T_{i, k} \leq C D_{i}^{L}\left(\lambda_{0}\right) \\
& C D_{i}^{L}\left(\lambda_{0}\right) \leq \sum_{k=1}^{N_{f}} C D_{i}^{L}\left(\lambda_{0}\right)_{k, \max } F T_{i, k}
\end{aligned}
$$

$C D_{i}^{L}\left(\lambda_{0}\right)_{k, \text { min }}$ and $C D_{i}^{L}\left(\lambda_{0}\right)_{k, \text { max }}$ represents respectively, the minimal and maximal possible value for the $i^{\text {th }}$ linkaccumulated chromatic dispersion if the link is $k$-type and due to the uncertainties on the fiber chromatic dispersions in data sheets $\left(\Delta D_{k}\right)$ and on link lengths $(\Delta L)$. They are expressed by Eq. (5)-(6):

$$
\begin{aligned}
& C D_{i}^{L}\left(\lambda_{0}\right)_{k, \text { min }}=\left(L_{i}-\Delta L\right)\left(D\left(\lambda_{0}\right)_{k}-\Delta D_{k}\right) \\
& C D_{i}^{L}\left(\lambda_{0}\right)_{k, \text { max }}=\left(L_{i}+\Delta L\right)\left(D\left(\lambda_{0}\right)_{k}+\Delta D_{k}\right)
\end{aligned}
$$

The following equations (7)-(10) are the equivalent of Eq. (3)-(6) for the link-accumulated chromatic dispersion slope $C D^{\prime}$ :

$$
\begin{aligned}
& \sum_{k=1}^{N_{f}} C D_{i}^{L}\left(\lambda_{0}\right)_{k, \min } F T_{i, k} \leq C D_{i}^{\prime L}\left(\lambda_{0}\right) \\
& C D_{i}^{\prime L}\left(\lambda_{0}\right) \leq \sum_{k=1}^{N_{f}} C D_{i}^{\prime L}\left(\lambda_{0}\right)_{k}, \max _{i} F T_{i, k} \\
& C D_{i}^{\prime L}\left(\lambda_{0}\right)_{k, \text { min }}=\left(L_{i}-\Delta L\right)\left(D^{\prime}\left(\lambda_{0}\right)_{k}-\Delta D_{k}^{\prime}\right) \\
& C D_{i}^{\prime L}\left(\lambda_{0}\right)_{k, \text { max }}=\left(L_{i}+\Delta L\right)\left(D^{\prime}\left(\lambda_{0}\right)_{k}+\Delta D_{k}^{\prime}\right)
\end{aligned}
$$

Equation (11) expresses that the fiber type is necessarily unique for a given link:

$$
\sum_{k=1}^{N_{f}} F T_{i, k}=1, F T_{i, k}=\{0,1\} .
$$

We propose a method based on mixed integer linear programming (MILP) to find the solution of the system of equations/inequalities (1)-(11) and estimate simultaneously all characteristics (fiber type, chromatic dispersion value and slope) of all links of the optical network as listed in the list of output parameters. Unfortunately, this system may have several solutions 


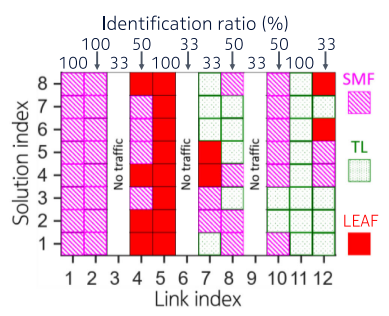

(a)

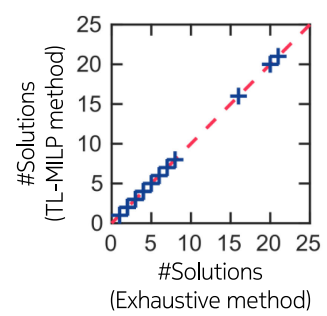

(b)

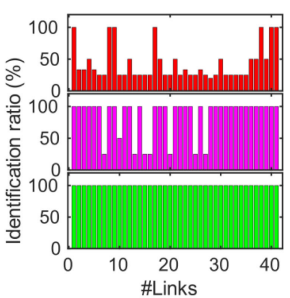

(c)

Fig. 2. (a) Example of all possible solutions (8) for 5 light paths in the 9-nodes network with 3 fiber types (from Fig. 1(c)) and a cumulated dispersion uncertainty of $200 \mathrm{ps} / \mathrm{nm}$ The identification ratio defines for each link the unambiguity of the identification. 100\%: unique solution, $50 \%$ : 2 possible fiber types, $33 \%$ : 3 possible fiber types. (b) Number of solutions for the complete network for 100 traffic matrices: Time Limited (TL)-MILP vs. exhaustive method. (c) Identification ratio for all links for three increasing numbers of LP in the 41-links network with 4 fiber types (Fig. 1b): 10 (top), 25 (middle) and 100 (bottom). 100\%: unique solution, 50\%: 2 possible fiber types, 33\%: 3 possible fiber types and 25\%: 4 possible fiber types.

when the system is under-defined (small number of light paths and/or with a high accumulated chromatic dispersion uncertainties). We use CPLEX software to find all possible solutions and obtain for each link of the network the set of all possible fiber types and the chromatic dispersion, simultaneously. The algorithm, based on a Branch-and-Bound method [19], is by default not exhaustive since it stops when one solution is found. A CPLEX method called "populate" can generate multiple solutions and the algorithm stops when the number of solutions reaches a certain user-defined threshold. Since the execution time is proportional to the number of requested solutions, we call the method Time-Limited MILP (TL-MILP).

\section{RESULTS}

\section{A. Topology}

In this work, we consider the European backbone network made of 28 nodes and $N=41$ bidirectional dispersion uncompensated links (Fig. 1(b)). The number next to each link corresponds to the link length as multiple of $100 \mathrm{~km}$. We assume that links are symmetric and have the same physical properties in both directions. We consider four fiber types and the chromatic dispersion characteristics are provided in Table I. They correspond to commercially available fiber types. TL is the TeraLight developed by Alcatel. For the DSF, we have chosen typical values for the dispersion and its slope. A network with an extreme heterogeneity of fiber type is rare but may occur as in the North American backbone topology used in [9]. It is not uncommon that link lengths also differ from operator's records [15]. Therefore, we emulate the link lengths with a random uniform distribution $\left(\mathrm{U}\left[L_{i}-\Delta L, L_{i}+\Delta L\right]\right)$ with $\Delta L=2 \mathrm{~km}$. For each fiber type, we assume that fiber chromatic dispersion $D$ and slope $D^{\prime}$ follow uniform distributions, $\mathrm{U}[D \Delta D, D+\Delta D]$ and $\mathrm{U}\left[D^{\prime}-\Delta D^{\prime}, D^{\prime}+\Delta D^{\prime}\right]$, respectively (see Table I). From the actual accumulated chromatic dispersion evaluated for each light path $j$ and wavelength $\lambda\left(C D_{j}^{L P}\right.$ actual $\left.(\lambda)\right)$, we emulate the corresponding measured values by modeling the $C D$ measurement uncertainty, $C D_{j}^{L P}$ meas $(\lambda)$, with a normal distribution $\mathcal{N}\left[C D_{j, \text { actual }}^{L P}(\lambda), \Delta D^{L P} / 6\right]$.

\section{B. Comparison With Exhaustive Search}

To make sure that the MILP-based method can find all solutions, we compare the solution pool with the one obtained with an exhaustive method. The MILP-based method stops as soon as the number of solutions reaches 20 . To obtain all solutions in an exhaustive way, we list all the possible arrangements of integer values for $N$ links knowing that each integer can take $N_{f}$ different values, where $N_{f}$ is the number of possible fiber types. For each arrangement, we solve the MILP with $N_{f}$ additional constraints. We obtain the number of exhaustive solutions by counting the number of times when only one solution is returned. Knowing that one MILP resolution takes around 1 second on our computer, it would take more than $4^{41}$ seconds i.e., $10^{17}$ years for the European network (41 links) and with 4 different fiber types. For a less time-consuming comparison, we choose a sub-part of this European network with only 9 nodes (represented by full squares in Fig. 1(c) and 3 different fiber types (SMF, TL and LEAF). The characteristics of these fibers are given in Table I.

For that network, we show in Fig. 2(a) all 8 possible solutions obtained with 5 established light paths and an uncertainty of $200 \mathrm{ps} / \mathrm{nm}$ on the light path accumulated chromatic dispersion. The system of equations/inequalities of the MILP is satisfied for all 8 combinations of fiber types. For each link, we quantify the amount of potential unambiguity of the fiber identification by defining the "identification ratio", which values appear at the top of the figure: $100 \%$ when the identified solution for the link is unique (no ambiguity), $50 \%$ when two possible values are possible for one link and 33\% when all 3 fiber types are possible. When there is no traffic on that link (shown in white on Fig. 2(a)), the identification ratio is equal to $33 \%$ since no fiber type can be distinguished among the three possibilities (full ambiguity).

To compare the two methods, we plot in Fig. 2(b) the number of solutions obtained with the TL-MILP method versus the exhaustive MILP-based method for 100 traffic matrices. Since all points are on the diagonal, the TL-MILP method can find, in few seconds, all possible solutions of the equations/inequalities system Eq. (1)-(11) as found by the exhaustive method.

\section{Identifying Four Fiber Types With Different Local Chromatic Dispersions}

After the validation of our TL-MILP method with a smaller size network, we identify four fiber types for the complete European network (28 nodes and 41 links) whose characteristics 


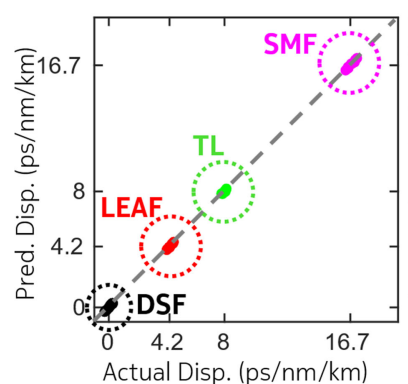

(a)

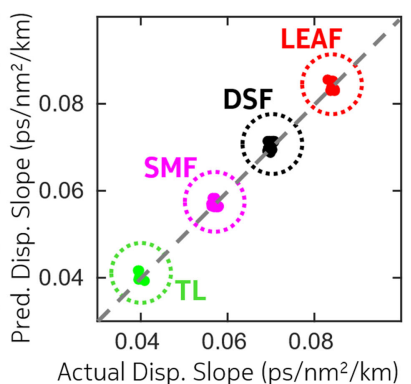

(b)
Fig. 3. (a) Predicted vs. actual dispersion for all network links. (b) Same with the dispersion slope.

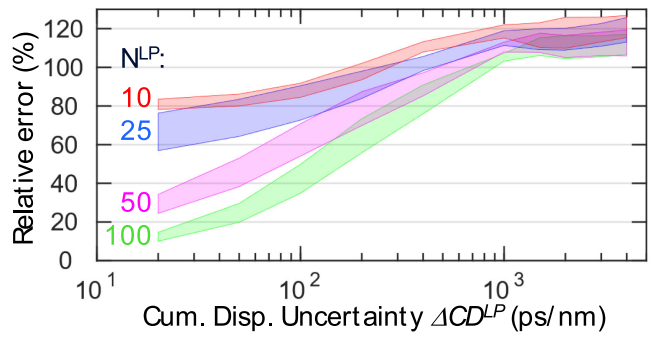

Fig. 4. Relative standard error of the chromatic dispersion values (with respect to the data sheet uncertainties) versus the uncertainty on the accumulated chromatic dispersion $\left(\triangle C D^{L P}\right)$ and for four numbers of light paths $\left(N^{L P}\right)$.

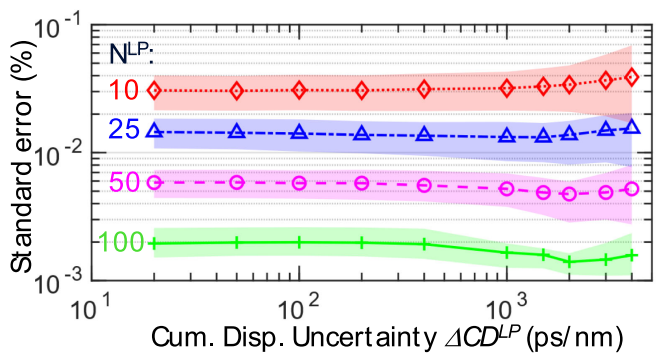

Fig. 5. Standard error of the chromatic dispersion values (with respect to the data sheet uncertainties) versus the uncertainty on the accumulated chromatic dispersion $\left(\triangle C D^{L P}\right)$ and for four numbers of light paths $\left(N^{L P}\right)$.

are shown in Table I. In Fig. 2(c) we show the identification ratio for a given network and for three increasing numbers of light paths, $N^{L P}: 10$ (top), 25 (middle) and 100 (bottom). With an increase of $N^{L P}$, we collect more information and we obtain strong improvement on the network knowledge as more links' fiber types are correctly identified. With $N^{L P}=100$, a unique solution is found for all links.

In Fig. 3(a) (resp. Fig. 3(b)), we plot the estimated $C D$ (resp. $C D$ slope) versus actual values for all links whenever a single solution to the problem is obtained $\left(N^{L P}=100\right)$. All points are close to the ideal estimation (i.e., along the diagonal), meaning that both dispersion and dispersion slope are well estimated. The average root-mean-square error of the dispersion estimation (resp. dispersion slope estimation root-mean-squared error) is plotted in Fig. 4 (resp. Fig. 5) as a function of the $C D$ uncertainty for $N^{L P}=\{10,25,50,100\}$. In Fig. 4, the error on the chromatic dispersion values has been normalized for each fiber type with respect to its own error specification (cf. Table I). The shaded area corresponds to the range of values obtained for the four fiber

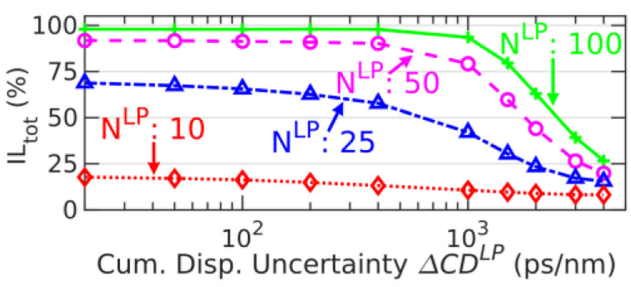

Fig. 6. Identification level $I L_{t o t}$ versus the uncertainty on the accumulated chromatic dispersion $\triangle C D^{L P}$ (measured) and for four number of light paths $\left(N^{L P}\right)$.

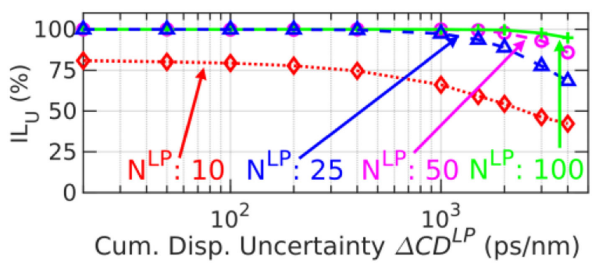

Fig. 7. Identification level $I L_{U}$ versus the uncertainty on the accumulated chromatic dispersion $\triangle C D^{L P}$ (measured) and for four number of light paths $\left(N^{L P}\right)$.

types. In Fig. 5, we plot the raw (not normalized) values since we consider the same uncertainty on the chromatic dispersion slopes for all fiber types. Together with the shaded area described for Fig. 4, we also add the error averaged over the four fiber types. The dispersion estimation error (Fig. 4) decreases with the number of light paths $N^{L P}$ and the $C D$ measurement uncertainty. By correlating enough accumulated dispersion measurements, we can reduce the uncertainty on the dispersion value by a factor of $10\left(N^{L P}=100\right.$ and $\left.\Delta C D^{L P}=20 \mathrm{ps} / \mathrm{nm}\right)$. By comparison, the error on the slope (Fig. 5) also decreases with $N^{L P}$ but is found to be almost independent of the $C D$ measurement accuracy and bounded by the small range of $C D$ slope in Table I.

In Fig. 2(c), we evaluate the number of solutions for all links but, even when there is a unique solution for one or all links, the quality of the identification is not necessarily guaranteed. In Fig. 6 and Fig. 7, we evaluate the number of correctly identified links and define two identification levels: the ratio of correctly identified links with the total number of links $\left(I L_{t o t}\right)$ or with the number of links for which a unique solution has been found $\left(I L_{U}\right)$ for the following network parameters:

- $N^{L P}$ : from 10 to 100 ;

- $C D$ uncertainty $\Delta \mathrm{C} D^{L P}$ : from 20 to $4000 \mathrm{ps} / \mathrm{nm}$;

- Uniform traffic matrix: 100 seeds;

- Fiber arrangements: 10 seeds;

- $C D$ measurement: 100 seeds.

Overall, Fig. 6 (resp. Fig. 7) shows the identification level $I L_{\text {tot }}\left(\right.$ resp. $\left.I L_{U}\right)$ averaged over all the $10^{5}$ runs and all links carrying traffic as a function of the $C D$ uncertainty for various $N^{L P}$ numbers. With only 100 LPs in 41-link network (13\% of the maximal traffic), the identification level $I L_{t o t}$ is higher than $98 \%$ for a $C D$ uncertainty up to $400 \mathrm{ps} / \mathrm{nm}$ when the typical measurement uncertainty is around $20 \mathrm{ps} / \mathrm{nm}$. For relatively low uncertainties (less than $400 \mathrm{ps} / \mathrm{nm}$ ) and $N^{L P}$ below 50, $I L_{t o t}$ decreases to unacceptably low level: less than 69\% (resp. 18\%) for $50 L P s$ (resp. $10 L P s$ ). Increasing uncertainties of the $C D$ 


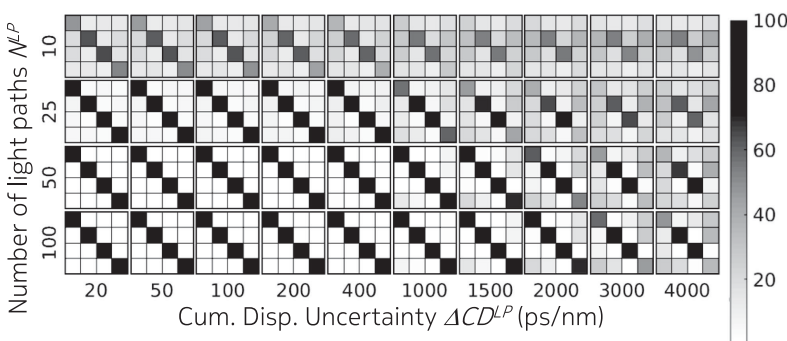

(a)

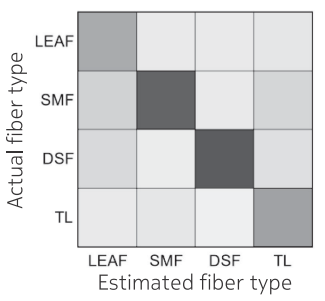

(b)

Fig. 8. (a) Confusion matrices for different number of light paths $N^{L P}$ and uncertainty on the accumulated chromatic dispersion $\triangle C D^{L P}$. (b) Zoom on a confusion matrix for $N^{L P}=10$ and an uncertainty on the accumulated chromatic dispersion $\Delta C D^{L P}$ of $200 \mathrm{ps} / \mathrm{nm}$.

uncertainty above $400 \mathrm{ps} / \mathrm{nm}$, particularly for less $50 L P s$, yields poor identification level $I L_{t o t}$.

$I L_{U}$ is the identification level for a given link even when a unique solution is found, see Fig. 7. For 50 and $100 L P s, I L_{U}$ is equal to $100 \%$ (perfect identification). Even when the fiber type identification is poor for the complete network (Fig. 6, $N^{L P}=$ 25 ), we can still have an identification level higher than $99.5 \%$ for links for which CPLEX returns a single solution and when $\Delta C D^{L P} \leq 400 \mathrm{ps} / \mathrm{nm}$. For $10 \mathrm{LPs}$ or for extremely large CD uncertainties, we have a very low identification level $\left(\mathrm{IL}_{\mathrm{U}}\right.$ of $80 \%$ and even less).

To illustrate this success/fail in the fiber type identification we build the confusion matrix for all the varying parameters: the number of light paths and the uncertainty on the measured accumulated chromatic dispersion, as displayed in Fig. 8(a). Fig. 8(b) represents an example of confusion matrix for $N^{L P}$ $=10$ and $\Delta C D^{L P}=200 \mathrm{ps} / \mathrm{nm}$. For all the $10^{5}$ runs and all links carrying traffic, we enumerate all the estimated fiber types for each actual value. A perfect fiber type identification would lead to a diagonal confusion matrix with full black squares. A confusion matrix with non-diagonal elements means that the identification level is lower than $100 \%$.

When there are more than two non-white elements in each column of the confusion matrix, several possible identifications are possible for a given actual fiber type. The SMF fiber can be confused with fibers which are not necessarily the closest in term of chromatic dispersion value (i.e., TL). The SMF fiber can be even confused with the fiber having the lowest chromatic dispersion value, the DSF fiber. This situation happens for large uncertainties on the accumulated dispersion and for small number of light paths. We can also see in Fig. 8 that the identification level is not the same for the four fiber types. The two middle elements (SMF, DSF) of the confusion matrix diagonal are generally darker than the other two elements (LEAF, TL) since SMF and DSF have less "neighbors" than the other two fibers.

\section{Identifying Five Fiber Types With Two Identical Local Chromatic Dispersions}

In the results presented in Section IV.C, the chromatic dispersions of all unknown fibers were different at least for the specification values. It can happen that two fibers have the same chromatic dispersion and differ only by their dispersion slope.

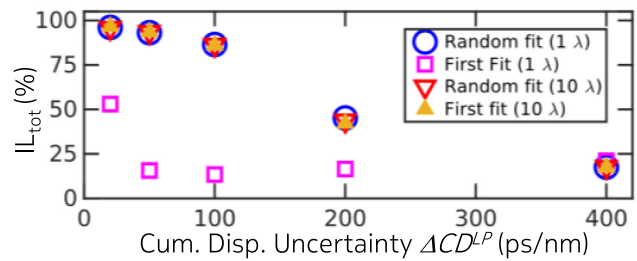

Fig. 9. Identification level $\mathrm{IL}_{\text {tot }}$ for a random and first fit wavelength allocation with 1 or 10 wavelengths per light path.

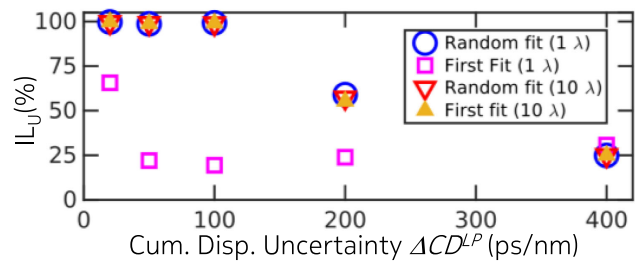

Fig. 10. Identification level $\mathrm{IL}_{\mathrm{U}}$ for a random and first fit wavelength allocation with 1 or 10 wavelengths per light path.

This case occurs for example with one LEAF fiber and the reduced slope true wave (TWRS) fiber from the manufacturer OFS (Optical Fiber Solutions). The chromatic dispersion is identical $(4.2 \mathrm{ps} / \mathrm{nm} / \mathrm{km})$ at $1550 \mathrm{~nm}$ but they have different dispersion slopes: $0.084 \mathrm{ps} / \mathrm{nm}^{2} / \mathrm{km}$ for the LEAF and $0.045 \mathrm{ps} / \mathrm{nm}^{2} / \mathrm{km}$ for the TWRS. To distinguish these two fiber types, we take advantage of the dispersion slope value which is also one of the outputs of our algorithm. The other fiber types are SMF, TL and DSF. In Fig. 9 and Fig. 10, we plot the two identification levels versus the accuracy of the accumulated chromatic dispersion measurement over the light path: $I L_{t o t}$ in Fig. 9 and $I L_{U}$ in Fig. 10.

Unlike in the study described in Section IV.C, we consider different wavelength allocations: 1 or 10 wavelengths allocated for each traffic demand with two allocation procedures, Random and First Fit. When comparing the obtained results with those presented in Fig. 6 and Fig. 7 for four different chromatic dispersion values, we can see that the identification levels are generally lower whatever the number of light paths. With the first fit wavelength allocation with one wavelength per light path and even for a very low light path accumulated chromatic dispersion uncertainty, both identification levels are very low: $I L_{t o t}=53 \%$ and $I L_{U}=65 \%$. With 10 wavelengths per light path, $97 \%$ of the network $\left(I L_{\mathrm{tot}}\right)$ is identified correctly with an identification 

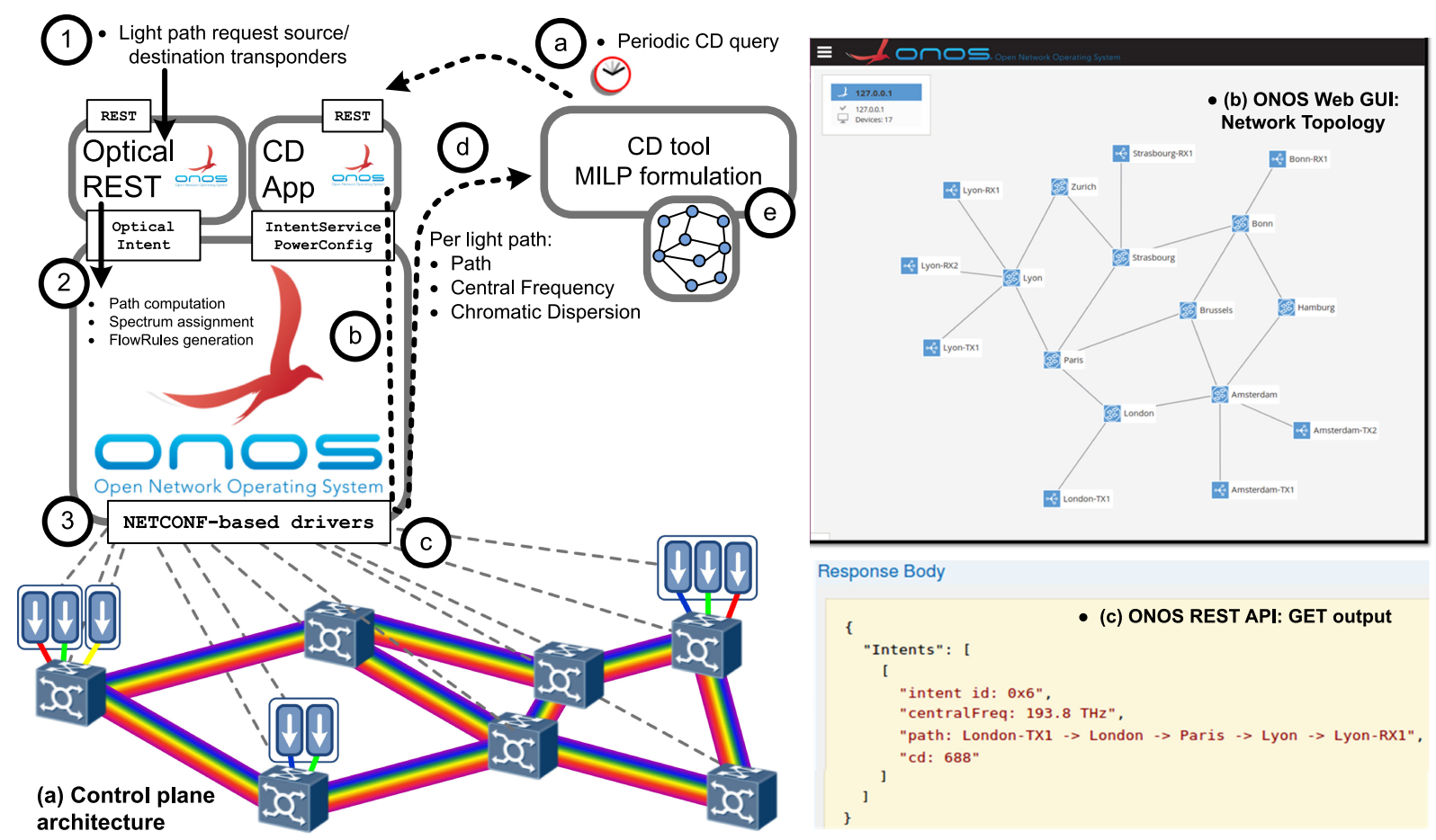

Fig. 11. (a) SDN control plane architecture, the Optical REST app is used to submit light path requests to ONOS, the CD app is used to process periodic requests by the external tool implementing the proposed MILP model within MATLAB. (b) Screenshot of the emulated network topology visualized on the ONOS web GUI. (c) Screenshot of the ONOS REST web API reporting information exchanged between ONOS and the MILP-based tool.

level of almost 100\% $\left(I L_{U}\right)$. The same estimation improvement is obtained with one wavelength per light path but with a random fit allocation procedure. Adding more wavelengths does not significantly improve the estimation accuracy. The edges of the C-band should be populated so that the chromatic dispersion of the LEAF and TWRS can sufficiently differ to remove this fiber ambiguity. This can be achieved by increasing the number of wavelengths per light path and/or by using a random fit allocation procedure. When we increase the uncertainty on the light path's accumulated chromatic dispersion, the possible range of values for the link accumulated chromatic dispersion increases in the same way and we rapidly get an overlap for the LEAF and TWRS fibers, leading to wrong fiber type identification. For an uncertainty of $200 \mathrm{ps} / \mathrm{nm}$, consider Fig. 10, the identification level $I L_{U}$ drops to less than $60 \%$ and only half of the network is correctly identified.

\section{SDN Control Plane Architecture AND LIVE DEMONSTRATION}

The technique described in the previous sections was demonstrated live at the ECOC 2018 conference [21]. The architecture of the deployed SDN control plane is depicted in Fig. 11. Specifically, the control plane integrates an ONOS controller with the proposed MILP-based tool through the utilization of a custombuilt ONOS application (i.e., CD App in Fig. 11); moreover, the Optical REST application (included in the official version 1.14 of ONOS) is utilized to establish/remove the light path on the optical data plane. This application exposes a REST interface to enable light path requests submission from external tools (e.g., network operator management software).

Fig. 11, also illustrates the procedure to activate a new light path. A new light path request is submitted from an external tool using a REST interface specifying the source and the destination transponders, step (1) in Fig. 11. Upon reception of the request, the Optical Intent feature of ONOS is used within the controller to perform routing and spectrum assignment, and determine all the FlowRules (i.e., the configurations required in each node of the data plane) to establish the light path, step (2) in Fig. 11. Once the FlowRules are generated, they are converted in NETCONF messages (e.g., <edit-config > messages) at the driver's level and sent to the devices, in accordance with the specific YANG model.

Fig. 11 also illustrates the interaction between the MILPbased tool implemented with MATLAB and the ONOS controller. The MILP-based tool periodically interrogates the controller to retrieve the information regarding active light paths needed for the fiber type identification, step (a) in Fig. 11. For this purpose, the CD App has been developed within ONOS exposing a REST API supporting the "GET" method to retrieve light paths data. Upon request from the MILP-based tool the CD App utilizes the IntentService to retrieve the information regarding currently established light paths (i.e., the path, the central frequency), step (b) in Fig. 11; moreover, the application utilizes the extended PowerConfig behavior of the NETCONF driver to retrieve the current dispersion value measured at the coherent receiver of each active light path, step (c) in Fig. 11. The collected data is replied to the MILP-based, step (d) in Fig. 11. Finally, Fig. 11 reports in the bottom-right corner a screenshot of the 


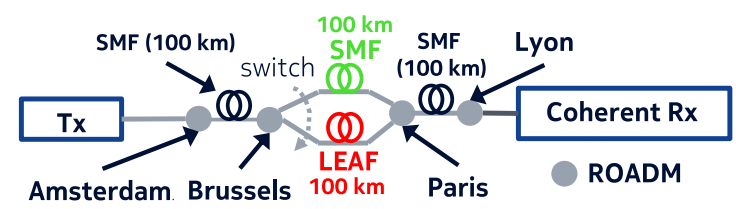

Fig. 12. Optical line system of the fiber type identification demonstration.

ONOS web interface reporting collected information regarding one active light path.

The described control plane solution was implemented and deployed in a live demonstration [21], using an emulated data plane including 9 nodes and 4 pairs of coherent transponders. The emulated topology is depicted with filled squares in Fig. 1 (c) and reported in the ONOS web GUI screenshot included in the top-right corner of Fig. 11. Optical nodes and transponders are emulated through the implementation of NETCONF agents representing the devices with the same YANG based models utilized in [22]. One of those agents is connected at a physical coherent transponder located in the Nokia labs near Paris. The "Lyon" transponder is the termination of the physical testbed illustrated in Fig. 12 which represents an optical line system from Amsterdam to Lyon, passing through two intermediate nodes: Brussels and Paris. Each fiber link is implemented with one spool of $100 \mathrm{~km}$ of fiber. The link between Brussels and Paris can use either SMF or LEAF fiber; the choice between those two types of fiber is made using an optical switch. One signal was transmitted over 32 GBaud PDM-QPSK modulated carrier at $1550 \mathrm{~nm}$. The real values of the dispersion measured at the transponder located at the Nokia labs are retrieved by the ONOS controller located at the TeCIP institute of Scuola Superiore Sant'Anna in Pisa.

During the demonstration in [21] the fiber type between Brussels and Paris is blindly selected by the visitor using a Android application, sending directly the fiber type choice to the switch. Then a sequence of four light paths were established on the emulated network, along the paths depicted in Fig. 11, with one light path terminating at the real Nokia transponder. Every time a new light path was established, the MILP-based tool requested light paths' data from the ONOS controller. When the fourth light path was established the MILP-tool had enough data to estimate the fiber type of the link between Brussels and Paris.

With the utilized version of ONOS, the time needed to establish a light path, including final confirmation of all performed configurations on the data plane was about 10-15 seconds, steps (1), (2) and (3) in Fig. 11. Conversely, the time required to retrieve the light path information including the communication with the data plane was about $100 \mathrm{~ms}$, steps (a), (b), (c) and (d) in Fig. 11.

\section{CONCLUSION}

By monitoring, centralizing and correlating the accumulated chromatic dispersion over all network light paths, we identify the fiber type for all unknown links of a European backbone network consisting of four types of fibers. Simultaneously, the chromatic dispersion and its slope are also assessed by MILPbased method and we automatically identify fiber type of all unknown links in 41-link/28-node network without traffic interruption or extra-cost and with only 100 light paths for an $98 \%$ identification level. Two fibers with the same chromatic dispersion, but different dispersion slope can be also distinguished. We have also implemented fiber type identification application with a real communication between the SDN controller and our Nokia labs for the ECOC'18 demo session [21].

\section{REFERENCES}

[1] E. Seve, C. Delezoide, J. Pesic, S. Bigo, and Y. Pointurier, “Automated fiber type identification,” in Proc. Eur. Conf. Opt. Commun., 2018, Paper Tu.3D.1.

[2] Y. Pointurier, "Design of low-margin optical networks," J. Opt. Commun. Netw., vol. 9, no. 1, pp. 9-17 2018.

[3] J-L. Augé, "Can we use flexible transponders to reduce margins?," in Proc. Opt. Fiber Commun. Conf. Expo. Nat. Fiber Opt. Eng. Conf., 2013, Paper OTu2A.1.

[4] E. Seve, J. Pesic, C. Delezoide, S. Bigo, and Y. Pointurier, "Learning process for reducing uncertainties on network parameters and design margins," IEEE/OSA J. Opt. Commun. Netw., vol. 10, no. 2, pp. A298-A306, Feb. 2018.

[5] P. Soumplis, K. Christodoulopoulos, M. Quagliotti, A. Pagano, and E. Varvarigos, "Network planning with actual margins," J. Lightw. Technol., vol. 35, no. 23, pp. 5105-5120, Dec.1, 2017.

[6] I. Sartzetakis, K. Christodoulopoulos, and E. Varvarigos, "Improving QoT estimation accuracy through active monitoring," in Proc. 19th Int. Conf. Transparent Opt. Netw., 2017, Paper We.B1.3.

[7] C. Rottondi, L. Barletta, A. Giusti, and M. Tornatore, "Machine-learning method for quality of transmission prediction of unestablished lightpaths," IEEE/OSA J. Opt. Commun. Netw., vol. 10, no. 2, pp. A286-A297, Feb. 2018.

[8] L. Barletta, A. Giusti, C. Rottondi, and M. Tornatore, "QoT estimation for unestablished lighpaths using machine learning," in Proc. Opt. Fiber Commun. Conf. Exhib., 2017, Paper Th. 1J1.

[9] J. Wass, J. Thrane, M. Piels, R. Jones, and D. Zibar, "Gaussian process regression for WDM system performance prediction," in Proc. Opt. Fiber Commun. Conf. Exhib., 2017, Paper Tu.3D7.

[10] M. Bouda et al., "Accurate prediction of quality of transmission based on a dynamically configurable optical impairment model," IEEE/OSA J. Opt. Commun. Netw., vol. 10, no 1, pp. 102-109 Jan. 2018.

[11] S. Oda et al., "Innovative optical networking by optical performance monitoring and learning process," in Proc. Eur. Conf. Opt. Commun., 2018, Paper Tu3D3.

[12] S. Oda, M. Bouda, O. Vassilieva, Y. Hirose, T. Hoshida, and T. Ikeuchi, "Network capacity improvement by quality of transmission estimator with learning process," in Proc. Eur. Conf. Opt. Commun., 2017.

[13] G. Liu et al., "The first testbed demonstration of cognitive end-to-end optical service provisioning with hierarchical learning across multiple autonomous systems" in Proc. Opt. Fiber Commun. Conf. Expo., 2018, Paper Th4D.7.

[14] S. Yan et al., "Field trial of machine-learning-assisted and SDN-based optical network planning with network scale monitoring database," in Proc. Eur. Conf. Opt. Commun., 2017, Paper Th.PDP.B.4.

[15] M. S. Zefreh and S. Asselin, "Capacity enhancement in optical networks using margin extraction," in Proc. Opt. Fiber Commun. Conf. Expo., 2018.

[16] E. Seve et al., "Semi-analytical model for the performance estimation of $100 \mathrm{~Gb} / \mathrm{s}$ PDM-QPSK optical transmission systems without inline dispersion compensation and mixed fiber types," in Proc. Eur. Conf. Opt. Commun., 2013, Paper Th. 1D2.

[17] J. Pesic, N. Rossi, and T. Zami, "Fiber type dependent benefits when deploying up-to-300GB/s elastic transponders adapting to ageing of margins," in Proc. Opt. Fiber Commun. Conf. Expo., 2018, Paper M.1A2.

[18] E. Torrengo et al., "Experimental validation of an analytical model for nonlinear propagation in uncompensated optical links," Opt. Exp., vol. 19, no. 26, pp. 790-798 2011.

[19] A. H. Land and A. G. Doig, "An automatic method for solving discrete programming problems," Econometrica, vol. 28, no. 3, pp. 497-520, 1960.

[20] [Online]. Available: http://sndlib.zib.de/home.action

[21] E. Seve et al., "Interactive automated fiber type identification," in Proc. Eur. Conf. Opt. Commun., 2018

[22] A. Sgambelluri et al., "Fully disaggregated ROADM white box with NETCONF/YANG control, telemetry, and machine learning-based monitoring," in Proc. Opt. Fiber Commun. Conf. Expo., 2018, Paper TuDS.9. 